\title{
Use of Mini Dental Implants for Management of a Deficient Ridge: A Case Report
}

\section{Mohamed chebil ${ }^{1}$, Yosra Gassara ${ }^{2}$, Mohamed Ben Khalifa ${ }^{4}$ and Dalenda Hadyaoui ${ }^{3}$ \\ ${ }^{1}$ Assistant Professor, Department of Fixed Prosthodontics, Academic Dental Clinic of Monastir, Tunisia}

${ }^{2}$ DDS, Department of Fixed Prosthodontics, Academic Dental Clinic of Monastir, Tunisia

${ }^{4}$ Professor, Head of Department of Dentistry Hospital Fattouma, Monastir, Tunisia

${ }^{3}$ Professor, Department of Fixed Prosthodontics, Academic Dental Clinic of Monastir, Tunisia

*Corresponding author: Dalenda Hadyaoui, Professor; Department of Fixed Prosthodontics, Academic Dental Clinic of Monastir, Tunisia, Email: dalendaresearch@gmail.com

\begin{abstract}
In the case of thin alveolar ridge, standard diameter implants can only be used after bone augmentation surgery. But not all of our patients are ready for this type of surgery. In some favorable situations where special precautions are taken, mini-implants can be an interesting alternative. Here, we report a clinical case of mini implant-supported rehabilitation to manage of a thin edentulous ridge.
\end{abstract}

Key words: Mini Dental Implant; Thin Alveolar Ridge; Implant-Supported Fixed Prosthesis

\section{Introduction}

Sufficient bone support is one of the essential requirements to indicate an implant-supported fixed prosthesis. Thin alveolar ridges can be encountered in our practice and there are different ways for their management. Surgical augmentation techniques allow the reconstruction of a bone thickness needed for the placement of a standard implant [1]. However, not all of our patients are ready for bone augmentation surgeries. Mini dental implant can be an interesting alternative, but special precautions must be taken [2]. The purpose of this article is to show, through a clinical case, the contribution of mini dental implant in the management of thin ridges in implant-supported fixed prosthesis.

\section{Case Report}

A 70-year-old female consulted the department of fixed Prosthodontics at the Dental Clinic of Monastir requesting for a fixed rehabilitation. The patient was in good general health condition. The clinical examination revealed a poorly developed oro-facial muscles, good oral hygiene. The vertical dimension of occlusion was preserved. The maxillary arch presented bilateral terminal edentulous ridges with the loss of the following teeth: 14, 15, 16, 17, 26 and 27 (Figure 1). 


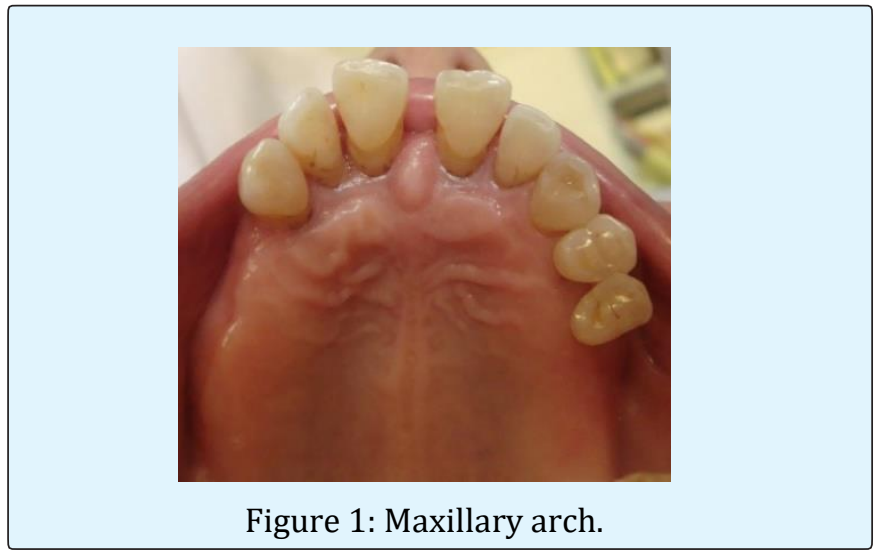

In the mandible, the teeth 46 and 36 were absent, metal retainers on teeth 34,35 and 37 were evaluated and found to be defectuous (Figure 2).

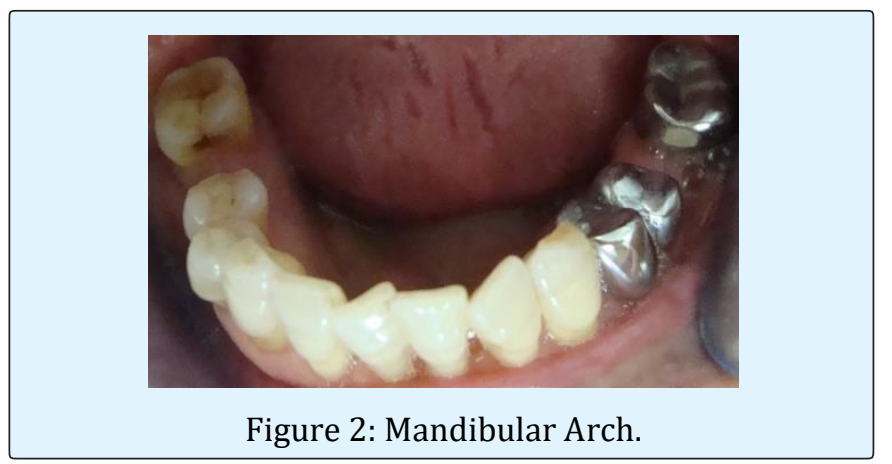

Examination of the maxillary and mandibular incisors showed slight tooth mobility. Examination of the occlusion showed a prosthetic occlusal height reduced on the right side due to the tooth 47egression (Figure 3).

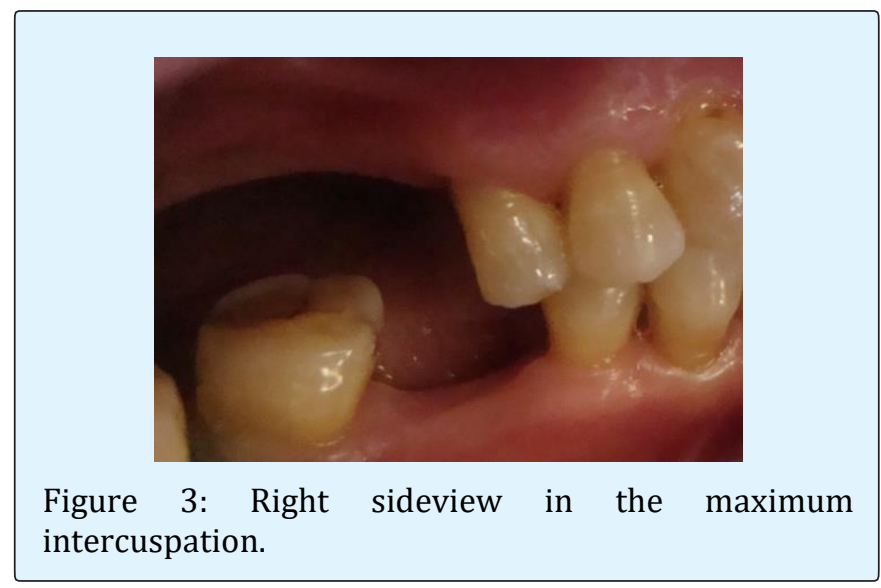

A panoramic film and periapical radiographs have revealed insufficient endodontic treatment on teeth 34 ,
35 and 37. A resorption of the right maxillary edentulous ridge was confirmed (Figure 4 ).

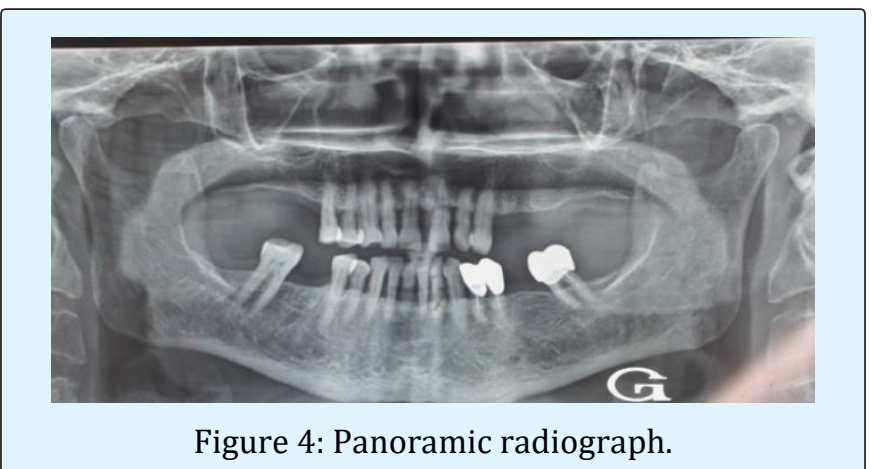

Diagnostic casts were mounted in centric occlusion then waxed to perform the prosthetic project. A bone volume study was achieved through a CT scan. We determined the different oblique slices passing through the edentulous maxillary sites. Then, we measured the thickness of the alveolar bone on each slide. The available bone height was considered in relation to the floor of the maxillary sinus. We found a thin alveolar crest thickness at the level of left premolar maxillary sector $(4.5 \mathrm{~mm})$. The available bone height was about of $15 \mathrm{~mm}$ (Figure 5).

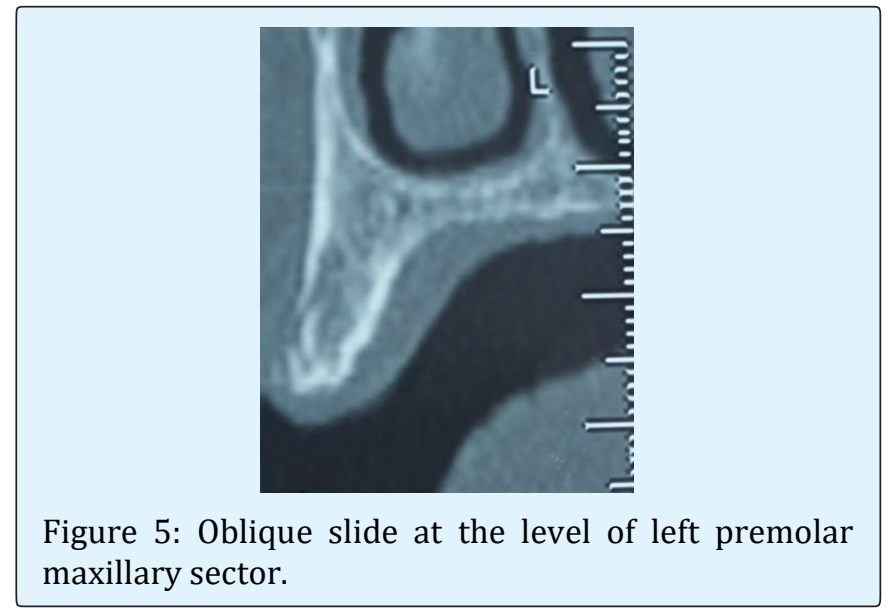

Our prosthetic decisions were:

- Two metal-ceramic crowns supported by two dental mini implants with a diameter $(\varnothing)$ of $2.5 \mathrm{~mm}$ and a length (L) of $13 \mathrm{~mm}$. in position of 24 and 25.

- Two metal-ceramic crowns supported by two implants with a diameter of $3,75 \mathrm{~mm}$ and a length of $11,5 \mathrm{mmin}$ position of 26 et 27

- A metal-ceramic implant-supported crown in position of 16 (Ø 3,75mm /L.8 mm).

- Two mandibular metal-ceramic bridges. 


\section{Medical Journal of Clinical Trials \& Case Studies}

\section{Preprosthetic Phase}

The main objective was the restoration of normal occlusal functions and the correction of posterior wedging. Loss this will remove occlusal overload on the anterior teeth. Provisional occlusal rehabilitation was performed using a maxillary removable partial denture and two temporary mandibular fixed partial dentures during this phase, endodontic restorations and posts and cores were performed.

\section{Surgical-Prosthetic Phase}

The placement of two mini dental implants in maxillary premolar region and three implants in molar regions was performed by the Flapless technique in one session (Figure 6).

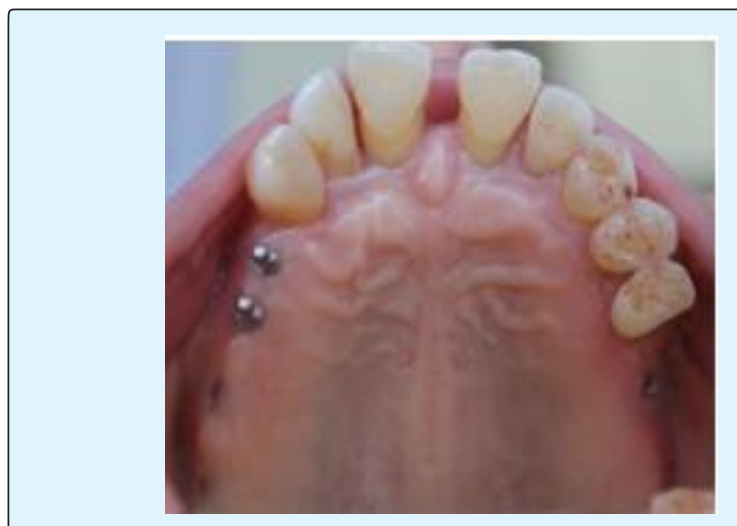

Figure 6: Occlusal view of the maxillary arch after implants placement.

For both mini dental implants, master impressions were performed after 10 weeks. In a first step, the metal abutments were sealed (Figure 7) and then the two metalceramic crowns. (Figure 8). Due care was given to excess removal of the cement to avoid any further complication.

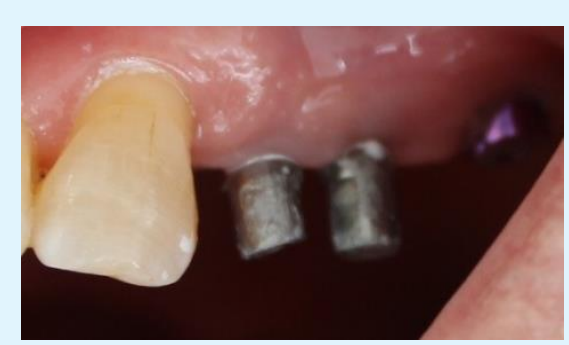

Figure 7: View of sealed abutments on mini dental implants.

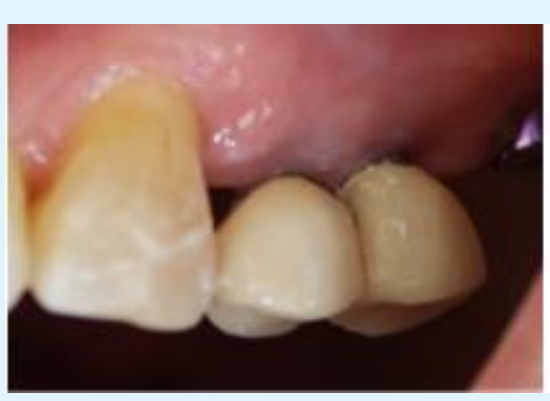

Figure 8: View of crowns sealed on the abutments.

Thereafter, we completed the implant-supported maxillary rehabilitation (Figure 9).

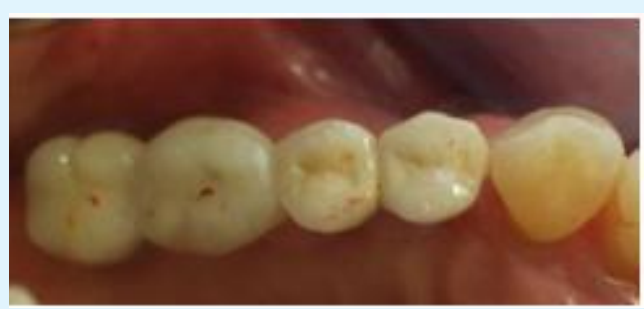

Figure 9: Occlusal view of the right maxillary rehabilitation.

In a later phase, the two temporary mandibular bridges were replaced by two metal-ceramic bridges (Figure 10).

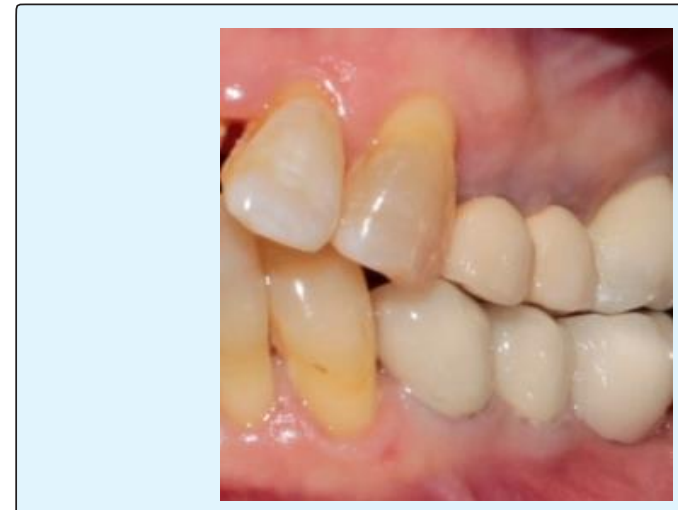

Figure 10: Left side view in maximum intercuspation.

\section{Discussion}

Our patient presented a thin alveolar ridge in the maxillary premolar area. Mini dental implants are indicated after a rigorous study. Specific precautions were taken. First, our patient had undeveloped masticatory muscles. This predicts that occlusal loads will be low. 


\section{Medical Journal of Clinical Trials \& Case Studies}

Moreover, the premolar areas are not as much solicited by occlusal forces as in the canine and molar sectors. Mini dental implants having a diameter of between 1.8 and $2.4 \mathrm{~mm}$ should be of sufficient length to optimize bone anchorage [3-5]. In our case, the thin bone crest had a height of $15 \mathrm{~mm}$, which allowed the placement of mini dental implants of sufficient length $(13 \mathrm{~mm})$. On the other hand, it is essential to obtain a good primary stability which will be, in part, provided by bone quality (preferably a type I and II bone) [5,6]. In our case, bone density was satisfactory. Mini dental implants had been inserted with the auto advance technique. Majority of mini-implants are composed of a titaniumalloy (Ti), Aluminum (Al) and Vanadium (V), making them more resistant to tensile forces than pure titanium [3,4].

Thus, they are sufficiently resistant to be inserted without prior preparation of the site $[7,8]$. The implant was inserted without reflecting the mucoperiosteal flap. It constitutes a minimally invasive technique and bone resorption will be reduced [9].

Mini dental implants have lower fracture resistance than standard implants, so specific control of transmitted occlusal loads is very important [10]. For this, we have previously restored normal occlusal conditions for our patient. This was done using temporary prostheses from the wax prosthetic project. The restoration of the posterior wedging as well as the distribution of the occlusal stresses is thus obtained. The reduction of occlusal surfaces has been the rule. In dynamic occlusion, our attention was focused on removing lateral forces at the level of mini implants, which will reduce the risk of fracture.

\section{Conclusion}

Mini dental implants are not intended to replace standard implants but represent a reliable alternative when conventional implantology cannot be considered for anatomic, medical or economic reasons. For the treatment of this case, all favorable conditions were met for the use of mini dental implants to manage the problem of thin ridge.

\section{References}

1. Schweizer P (2006) Intérêts des mini-implants. Information Dentaire 40: 2583-2586.

2. Christensen GJ (2006) The 'mini'-implant has arrived. J Am Dent Assoc 137(3): 387-390.

3. Shatkin TE, Shatkin S, Oppenheimer BD, Oppenheimer AJ (2007) Mini dental implants for long term fixed and removable prosthetics: a retrospective analysis of 2514 implants placed over a five year period. Compend Contin Educ Dent 28(2): 92-99.

4. Kheur M (2011) Transitional implants: An asset to implantology. J Interdiscip Dentistry 1(1): 4-9.

5. Davarpanah M, Szmukler-moncler S (2008) Manuel d'implantologie clinique: concepts, protocoles et innovations récentes. Paris CdP, pp: 539.

6. LemaYB (2010) Mini-dental implants: as even year clinical follow up. Journal of Canadian Denturism pp: 19-25.

7. Kanie T, Nagata M, Ban S (2004) Comparison of the mechanical properties of 2 prosthetic mini-implants. Implant Dent 13(3): 251-256.

8. Balkin BE, Steflid DE, Naval F (2001) Mini dental implant insertion with the auto advance technique for ongoing applications. J Oral Implantol 27(1): 32-37.

9. Job S, Bhat V (2008) An insight into flapless implant placement technique. J Indian Prosthodont Soc 8(3): 140-143.

10. Allum SR, Tomlinson RA, Joshi R (2008) The impact of loads on standard diameter, small diameter and mini implants: a comparative laboratory study. Clinical Oral Implants Res 19(6): 553-559.

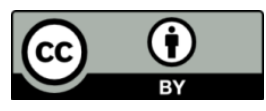

Dalenda Hadyaoui, et al. Use of Mini Dental Implants for Management of a Deficient Ridge: A Case Report. Med J Clin Trials Case Stud 2019, 3(1): 000197. 